\title{
The Calculated Genetic Barrier for Antiretroviral Drug Resistance Substitutions Is Largely Similar for Different HIV-1 Subtypes
}

\author{
David A. van de Vijver,* Annemarie M. J. Wensing,*† Gioacchino Angarano, $\neq$ \\ Birgitta Asjö, \$ Claudia Balotta,// Enzo Boeri, 9 Ricardo Camacho,\# Marie-Laure Chaix,** \\ Dominique Costagliola, $\uparrow$ Andrea De Luca, + Inge Derdelinckx, $\$$ Zehava Grossman,/// \\ Osamah Hamouda, 99 Angelos Hatzakis, \#\# Robert Hemmer, *** Andy Hoepelman, $\dagger$ Andrzej Horban, $\dagger+\uparrow$ \\ Klaus Korn, $+1+$ Claudia Kücherer, 99 Thomas Leitner, $\$ \$$ Clive Loveday, //I// Eilidh MacRae,//I// \\ Irina Maljkovic, 199 Carmen de Mendoza,\#\#\# Laurence Meyer,**** Claus Nielsen, + $+\dagger \dagger$ \\ Eline L. M. Op de Coul, $+1 \neq$ Vidar Ormaasen, $\$ \oint \xi$ Dimitris Paraskevis,\#\# Luc Perrin,//I/I/I \\ Elisabeth Puchhammer-Stöckl, $\$$ 9 9 9 Lidia Ruiz,\#\#\#\# Mika Salminen, ***** Jean-Claude Schmit,*** \\ Francois Schneider,*** Rob Schuurman,* Vincent Soriano,\#\#\# Grzegorz Stanczak, $\uparrow+\uparrow$ \\ Maja Stanojevic, $+\dagger \dagger+$ Anne-Mieke Vandamme, $\S$ Kristel Van Laethem, $\$$ Michela Violin,// \\ Karin Wilbe, 999 Sabine Yerly, ///I/// Maurizio Zazzi,t+1+t \\ and Charles A. B. Boucher, ${ }^{*}$ on behalf of the SPREAD Programme
}

Background: The genetic barrier, defined as the number of mutations required to overcome drug-selective pressure, is an important factor for the development of HIV drug resistance. Because of high variability between subtypes, particular HIV-1 subtypes could have different genetic barriers for drug resistance substitutions. This study

From the *Eijkman Winkler Institute, Department of Virology, University Medical Center Utrecht, Utrecht, the Netherlands; †Department of Internal Medicine, University Medical Center Utrecht, Utrecht, the Netherlands; †University of Foggia, Foggia, Italy; §University of Bergen, Bergen, Norway; \|University of Milan, Milan, Italy; qDiagnostica e Ricerca San Raffaele, Milan, Italy; \#Hospital Egas Moniz, Lisbon, Portugal; **Laboratoire de virologie, Hôpital Necker Paris, France; $\dagger+$ INSERM EMI 0214, CHU Pitié-Salpétrière, Paris, France; \$†Institute of Clinical Infectious Diseases, Catholic University, Rome, Italy; §§Rega Institute for Medical research, Katholieke Universiteit Leuven, Leuven, Belgium; ||||Sheba Medical Center, Tel-Hashomer, Israel; qףRobert Koch Institute, Berlin, Germany; \#\#Athens University Medical School, Athens, Greece; ***Centre Hospitalier de Luxembourg, Luxembourg; $\dagger \dagger+$ Hospital for Infectious Diseases \& AIDS Diagnosis and Therapy Center, Warsaw, Poland; + t+University of Erlangen, Erlangen, Germany; $\S \S \S$ Los Alamos National Laboratory, Los Alamos, USA; ||||||International Clinical Virology Centre, Buckinghamshire, England, United Kingdom; 919 Swedish Institute for Infectious Disease Control, Solna, Sweden; \#\#\#Hospital Carlos III, Madrid, Spain; ****INSERM U569, KremlinBicêtre, France; ††† †Statens Serum Institute, Copenhagen, Denmark; It+National Institute for Public Health and the Environment, Bilthoven, the Netherlands; $\S \S \S \S$ Ullevaal University Hospital, Oslo, Norway;

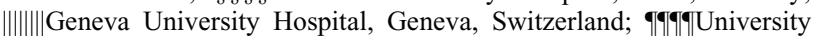
of Vienna, Vienna, Austria; \#\#\#\#Retrovirology Laboratory IRSICAIXA Foundation, Badalona, Spain; *****National Public Health Institute, Helsinki, Finland; †† Montenegro; and $+4+t+$ University of Siena, Siena, Italy.

Reprints: Charles Boucher Department of Virology, University Medical Center Utrecht, G04-614, 3584 CX Utrecht, the Netherlands (e-mail: C.Boucher@azu.nl).

Copyright (C) 2006 by Lippincott Williams \& Wilkins compared the genetic barrier between subtypes using some 2000 HIV-1 sequences ( $>600$ of non-B subtype) isolated from antiretroviral-naive patients in Europe.

Methods: The genetic barrier was calculated as the sum of transitions (scored as 1) and/or transversions (2.5) required for evolution to any major drug resistance substitution. In addition, the number of minor protease substitutions was determined for every subtype.

Results: Few dissimilarities were found. An increased genetic barrier was calculated for I82A (subtypes C and G), V108I (subtype G), V118I (subtype G), Q151M (subtypes D and F), L210W (subtypes C, F, G, and CRF02_AG), and P225H (subtype A) $(P<0.001$ compared with subtype B). A decreased genetic barrier was found for I82T (subtypes C and G) and V106M (subtype C) $(P<0.001$ vs subtype B). Conversely, minor protease substitutions differed extensively between subtypes.

Conclusions: Based on the calculated genetic barrier, the rate of drug resistance development may be similar for different HIV-1 subtypes. Because of differences in minor protease substitutions, protease inhibitor resistance could be enhanced in particular subtypes once the relevant major substitutions are selected.

Key Words: HIV, non-B subtypes, drug resistance, genetic barrier (J Acquir Immune Defic Syndr 2006;41:352-360)

L IV drug resistance is the consequence of mutations that are selected in the viral genes targeted by antiretroviral drugs. The genetic barrier, defined as the number of viral mutations required to overcome the drug-selective pressure, is therefore an important factor for the development of drug resistance. ${ }^{1,2}$ Boosted protease inhibitors have in general a high genetic barrier as they often require the accumulation 
of several mutations before the virus can overcome the selected drug pressure. Conversely, non-nucleoside reverse transcription inhibitors (NNRTIs) have a low genetic barrier as development of resistance can occur after only a single amino acid substitution. ${ }^{1}$

The huge variability between subtypes at the nucleotide level could have a significant impact on the genetic barrier for drug resistance. This impact of inter-subtype genetic variability is nicely illustrated by the V106M substitution, which is selected for under efavirenz exposure. Despite widespread efavirenz therapy in subtype B-infected patients, this substitution was not identified until studies in subtype $\mathrm{C}$ were performed. The increased propensity for subtype $\mathrm{C}$ to acquire $\mathrm{V} 106 \mathrm{M}$ is due to an alternate valine codon (GTG) compared with subtype B (GTA). The genetic barrier is smaller in subtype $\mathrm{C}$, as the number of mutations required to acquire $\mathrm{V} 106 \mathrm{M}$ is reduced from 2 in subtype $\mathrm{B}$ ( $G \mathrm{~T} A$ to $A \mathrm{~T} G$ ) to 1 in subtype $\mathrm{C}$ ( $G \mathrm{TG}$ to $A \mathrm{TG}) .^{3-5}$

Currently, due to the limited availability of sequences other than subtype B, it is not fully understood to what extent genetic differences between subtypes affect drug resistance patterns. Understanding the impact of genetic subtype variation on drug resistance is of utmost importance because an increasing number of patients infected with a non-B subtype virus are treated with antiretroviral drugs. To assess differences in the genetic barrier for evolution of drug resistance substitutions between subtypes, we compared over 600 non-B and more than 1300 subtype B sequences obtained from anti-retroviral-naive patients. ${ }^{6}$

\section{METHODS}

\section{Study Population}

The present study included samples from the CATCH study (Combined Analysis of the Resistance Transmission Over Time of Chronically and Acute Infected HIV Patients in Europe), ${ }^{6}$ which is part of the official European Commission supported scientific surveillance program SPREAD. The CATCH study was also open to centers currently not participating in the SPREAD program. HIV-1 seropositive individuals were eligible for this study if they had never been exposed to antiretroviral drugs before the time of sampling. In total, 2208 patients were included during 1996-2002 from the following 19 countries (no. of patients): Austria (84), Belgium (128), Denmark (116), Finland (8), France (249), Germany (62), Greece (40), Israel (104), Italy (365), Luxembourg (161), the Netherlands (25), Norway (23), Poland (35), Portugal (91), Serbia and Montenegro (10), Spain (142), Sweden (153), Switzerland (260), and the United Kingdom (152). ${ }^{6}$ For each patient, a single HIV-1 sequence was included.

\section{Genotypic Resistance Analysis}

Nucleotide sequence analysis of the HIV pol gene was performed in the participating centers using their own individual protocols. To assure the quality of the data, each submitted sequence was checked before inclusion. Sequences that contained stop codons and individual resistance codons with ambiguities consisting of $>2$ bases per nucleotide position or of $>2$ ambiguities per codon were excluded from the analysis. ${ }^{6}$ The protease sequences contained at least positions 10-90 and the reverse transcription (RT) sequences contained at least codons 41-220. The sequences from France included the RT region only.

Subtypes were compared for evolution to the drugresistance-associated substitutions specified by the International AIDS-Society (IAS)-USA, update of $2005 .^{7}$ In the protease region, the IAS distinguishes between major and minor substitutions. The major substitutions by themselves reduce drug susceptibility. Minor substitutions improve, in some cases, the replicative capacity of HIV carrying major substitutions, ${ }^{8}$ but by themselves they do not have a significant effect on drug susceptibility. ${ }^{7,8}$ Minor protease substitutions are also common in sequences that have not been exposed to antiretrovirals. ${ }^{9,10}$ The IAS-USA list does not include several substitutions important for tipranavir resistance. Therefore, for tipranavir L10V, I13V, K20M/R/V, L33F, E35G, M36I, K43T, M46L, I47V, I54A/M/V, Q58E, H69K, T74P, V82L/T, and I84V were included.

Sequences already containing major drug resistance substitutions were excluded in this study.

\section{Subtype Classification}

The genetic subtype of the viruses was assessed by means of phylogenetic analysis of pol sequences. The neighbor-joining method was used to compare the sequences to reference strains of known subtype derived from the Los Alamos database (www.hiv.lanl.gov). Pairwise distance matrices were generated using the Kimura 2-parameter distance estimation method with a transition/transversion ratio of 2.0. The consistency of the phylogenetic clustering was tested using bootstrap analysis with 100 replicates. Bootstrap values above 70 were considered sufficient for subtype assignment. ${ }^{11}$

\section{Calculated Genetic Barrier for Major Resistance Substitutions}

The genetic barrier will be influenced by the number and type of nucleotide mutations (ie, transitions and transversions) required for evolution from a wild-type codon to a major drugresistance-associated substitution. The type of nucleotide mutation influences the genetic barrier as transitions (the replacement of a purine by another purine: $\mathrm{A} \leftrightarrows \mathrm{G}$; or of a pyrimidine by another pyrimidine: $\mathrm{C} \leftrightarrows \mathrm{T}$ ) are for steric reasons occurring on average 2.5 more frequently (A.-M. Vandamme, Rega Institute for Medical research, Katholieke Universiteit Leuven, Belgium, personal communication) than transversions (the replacement of a purine by a pyrimidine and vice versa: $\mathrm{A} \leftrightarrows \mathrm{C}, \mathrm{A} \leftrightarrows \mathrm{T}, \mathrm{G} \leftrightarrows \mathrm{C}, \mathrm{G} \leftrightarrows \mathrm{T}){ }^{12}$ The genetic barrier was calculated for every individual sequence at all major drug-resistance-associated substitutions. To calculate the genetic barrier, the smallest number of transitions (scored as 1) and/or transversions (scored as 2.5) required for evolution to a drug-resistance-associated substitutions was determined. The calculated genetic barrier was taken as the sum of the scores for a particular drug resistance substitution. 
TABLE 1. Baseline Characteristics

\begin{tabular}{|c|c|c|}
\hline & Protease & RT \\
\hline Number & $1855^{*}$ & $1942 \dagger$ \\
\hline Age (SD) & $36(10)$ & $36(10)$ \\
\hline Male $(\%)$ & 73 & 73 \\
\hline \multicolumn{3}{|l|}{ Route of transmission $(\%)$} \\
\hline MSM & 41 & 42 \\
\hline Heterosexual & 41 & 41 \\
\hline IDU & 17 & 15 \\
\hline Other & 2 & 2 \\
\hline CD4, median (range), cells $/ \mathrm{mm}^{3}$ & $392(1-1764)$ & $405(1-1764)$ \\
\hline HIV-RNA load, mean (SD), log copies/mL & $4.77(0.82)$ & $4.83(0.80)$ \\
\hline \multicolumn{3}{|c|}{ Duration of infection $(\%)$} \\
\hline$<1$ year & 27 & 34 \\
\hline$>1$ year & 31 & 28 \\
\hline Unknown & 42 & 38 \\
\hline \multicolumn{3}{|c|}{$\begin{array}{l}\text { *Excluded were } 353 \text { patients from the original } 2208 \text { because they came from France } \\
(\mathrm{n}=249 \text {, no French protease sequences were available), carried at least } 1 \text { resistance- } \\
\text { associated major protease mutation }(\mathrm{n}=54) \text {, the HIV-1 protease sequence had missing } \\
\text { codons for positions } 10-90(\mathrm{n}=30) \text {, or had an un-typeable or a too rare subtype }(\mathrm{n}=36) \text {. } \\
\text { Numbers do not add up, as a patient could have met several exclusion criteria. } \\
\quad \dagger \text { Excluded were } 266 \text { patients, carried at least } 1 \text { NRTI resistance-associated mutation } \\
(\mathrm{n}=165) \text { or NNRTI resistance-associated mutation }(\mathrm{n}=64) \text {, the HIV-1 RT sequences } \\
\text { contained missing codons for any of the positions } 41-220(\mathrm{n}=95) \text {, or had an un- } \\
\text { typeable or a too rare subtype }(\mathrm{n}=36) \text {. Numbers do not add up, as a patient could have } \\
\text { met several exclusion criteria. }\end{array}$} \\
\hline
\end{tabular}

\section{Comparison of Minor Protease Resistance Substitutions}

The number of minor protease substitutions present in every individual sequence was determined for all protease inhibitors, and averaged for each subtype. The ratio of the mean numbers of a particular subtype and subtype B was then calculated. A value of $<1$ indicated a smaller number of minor substitutions as compared with subtype $\mathrm{B} ;>1$ means a higher number.

\section{Statistical Analysis}

Statistical tests were performed using all sequences from non-B subtype and a random selection of $20 \%$ of the subtype B sequences. Kruskal-Wallis tests were used for comparing the calculated genetic barrier for major substitutions and the number of minor protease substitutions between subtypes. When a significant value occurred $(P<$ 0.05), the pairwise difference between subtypes $\mathrm{B}$ and a particular non-B subtype was analyzed using the MannWhitney $U$ test and the Benjamini-Hochberg method (using a false-discovery rate of 0.01 ) to correct for multiple hypothesis testing. ${ }^{13}$

\section{RESULTS}

\section{Distribution of Subtypes}

The present study included 1855 protease and 1942 RT sequences for which a subtype could be assessed (Table 1). The sequences that were excluded because they contained any major drug-resistance-associated substitutions were more likely to be of subtype $B$ and to be isolated from patients that were infected for $<1$ year. ${ }^{6}$ Identified were subtypes A, B, C, D, F, G, J, CRF01_AE, and CRF02_AG (Tables 2, Tables 3 Tables 4). The most common subtype was B, with 1299

TABLE 2. Prevalence of Wild-Type (WT) Codons and Its Impact on Calculated Genetic Barrier at Major Protease Drug-ResistanceAssociated Positions in Anti-Retroviral-Naive Patients

\begin{tabular}{|c|c|c|c|c|c|c|c|c|c|c|c|c|c|c|}
\hline \multirow{2}{*}{\multicolumn{2}{|c|}{ Position }} & \multirow[b]{2}{*}{$\begin{array}{c}\text { WT } \\
\text { Codon }\end{array}$} & \multicolumn{9}{|c|}{ Proportion (\%) WT Codon by HIV-1 Subtype } & \multirow{2}{*}{$\begin{array}{c}\text { Closest } \\
\text { Mutational- } \\
\text { Resistant } \\
\text { Codon }\end{array}$} & \multirow[b]{2}{*}{$\begin{array}{c}\text { Required } \\
\text { Substitution* }\end{array}$} & \multirow[b]{2}{*}{$P \dagger$} \\
\hline & & & $\begin{array}{c}A \\
(n=68)\end{array}$ & $\begin{array}{c}B \\
(n=1299)\end{array}$ & $\begin{array}{c}C \\
(n=209)\end{array}$ & $\begin{array}{c}D \\
(n=24)\end{array}$ & $\begin{array}{c}F \\
(n=26)\end{array}$ & $\begin{array}{c}G \\
(n=86)\end{array}$ & $\begin{array}{c}J \\
(n=20)\end{array}$ & $\begin{array}{c}\text { CRF_AE } \\
(n=52)\end{array}$ & $\begin{array}{c}\text { CRF_AG } \\
(\mathrm{n}=71)\end{array}$ & & & \\
\hline \multirow[t]{2}{*}{30} & D30N & GAT & 100 & 97 & 94 & 98 & 100 & 92 & 100 & 98 & 93 & AAT & 1 ts & 1.00 \\
\hline & & GAC & - & 1 & 3 & - & - & 8 & - & - & 3 & AAC & $1 \mathrm{ts}$ & \\
\hline \multirow[t]{2}{*}{46} & M46I & ATG & 100 & 100 & 100 & 100 & 100 & 100 & 100 & 100 & 100 & ATA & 1 ts & 1.00 \\
\hline & M46L & ATG & & & & & & & & & & C/TTG & $1 \mathrm{tv}$ & 1.00 \\
\hline \multirow[t]{2}{*}{48} & G48V & GGG & 96 & 96 & 15 & 96 & 96 & 94 & 95 & 98 & 99 & GTG & $1 \mathrm{tv}$ & 1.00 \\
\hline & & GGA & 4 & 4 & 81 & 4 & 4 & 5 & 5 & 2 & 1 & GTA & $1 \mathrm{tv}$ & \\
\hline \multirow[t]{2}{*}{50} & I50L & ATT & 99 & 100 & 100 & 100 & 100 & 99 & 100 & 100 & 99 & CTT & $1 \mathrm{tv}$ & 0.20 \\
\hline & $\mathrm{I} 50 \mathrm{~V}$ & ATT & & & & & & & & & & GTT & 1 ts & 0.20 \\
\hline \multirow[t]{8}{*}{82} & V82A & GTC & 91 & 96 & 87 & 92 & 100 & 14 & 95 & 92 & 92 & $\mathrm{GCC}$ & 1 ts & $<0.001$ \\
\hline & $\mathrm{I} 82 \mathrm{~A}$ & ATC & - & 1 & 9 & 4 & - & 84 & 5 & 4 & 4 & GCC & 2 ts & \\
\hline & V82F & GTC & & & & & & & & & & TTC & $1 \mathrm{tv}$ & 0.92 \\
\hline & $\mathrm{I} 82 \mathrm{~F}$ & ATC & & & & & & & & & & TTC & $1 \mathrm{tv}$ & \\
\hline & V82S & GTC & & & & & & & & & & TCC & 1 ts & 1.00 \\
\hline & I82S & ATC & & & & & & & & & & TCC & 1 ts & \\
\hline & V82T & GTC & & & & & & & & & & $\mathrm{ACC}$ & 2 ts & $<0.001$ \\
\hline & $\mathrm{I} 82 \mathrm{~T}$ & ATC & & & & & & & & & & $\mathrm{ACC}$ & 1 ts & \\
\hline 84 & I84V & ATA & 100 & 100 & 100 & 100 & 100 & 100 & 100 & 100 & 100 & GTA & $1 \mathrm{ts}$ & 1.00 \\
\hline 90 & L90M & TTG & 100 & 97 & 96 & 96 & 100 & 97 & 100 & 98 & 100 & ATG & $1 \mathrm{tv}$ & 0.47 \\
\hline
\end{tabular}

*ts indicates transition; tv, transversion. The numbers designate the number of transitions/transversions required.

$\dagger P$ value calculated using the Kruskal-Wallis test and a value of 2.5 for a transversion and 1 for a transition. For statistical reasons, a random sample of $20 \%$ of the subtype $\mathrm{B}$ and all sequences classified with a subtype other than B were included in the Kruskal-Wallis test. 
TABLE 3. Prevalence of Wild-Type (WT) Codons and Its Impact on Calculated Genetic Barrier at NRTI Drug-Resistance-Associated Positions in Anti-Retroviral-Naive Patients

\begin{tabular}{|c|c|c|c|c|c|c|c|c|c|c|c|c|c|c|}
\hline \multirow[b]{2}{*}{ Codon } & \multirow[b]{2}{*}{ Substitution } & \multirow[b]{2}{*}{$\begin{array}{c}\text { WT } \\
\text { Codon }\end{array}$} & \multicolumn{10}{|c|}{ Proportion (\%) WT Codon by HIV-1 Subtype } & \multirow[b]{2}{*}{ Mutation* } & \multirow[b]{2}{*}{$P \dagger$} \\
\hline & & & $\begin{array}{c}\mathbf{A} \\
(\mathbf{7 3}) \ddagger\end{array}$ & $\begin{array}{c}\text { B } \\
(1338)\end{array}$ & $\begin{array}{c}\mathrm{C} \\
(213)\end{array}$ & $\begin{array}{c}\text { D } \\
(27)\end{array}$ & $\begin{array}{c}\mathbf{F} \\
(\mathbf{3 0}) \\
\end{array}$ & $\begin{array}{c}G \\
(89)\end{array}$ & $\begin{array}{c}\mathbf{J} \\
(27) \\
\end{array}$ & $\begin{array}{l}\mathbf{A E} \S \\
(55)\end{array}$ & $\begin{array}{l}\mathbf{A G \|} \| \\
(\mathbf{9 0})\end{array}$ & $\begin{array}{c}\text { resistant } \\
\text { codon }\end{array}$ & & \\
\hline 41 & M41L & ATG & 99 & 100 & 99 & 100 & 97 & 100 & 96 & 98 & 99 & $\mathrm{C} / \mathrm{TTG}$ & $1 \mathrm{tv}$ & 0.15 \\
\hline \multirow[t]{2}{*}{44} & E44D & GAA & 99 & 98 & 97 & 100 & 93 & 100 & 100 & 100 & 90 & $\mathrm{GAC} / \mathrm{T}$ & $1 \mathrm{tv}$ & 0.92 \\
\hline & & GAG & 1 & 1 & 2 & - & 3 & - & - & - & 7 & $\mathrm{GAC} / \mathrm{T}$ & $1 \mathrm{tv}$ & \\
\hline \multirow[t]{3}{*}{62} & A62V & GCC & 4 & 86 & 90 & 67 & 93 & 91 & 82 & 2 & 90 & GTC & 1 ts & 0.97 \\
\hline & & GCT & 84 & 8 & 6 & 30 & 7 & 9 & 11 & 98 & 9 & GTT & $1 \mathrm{ts}$ & \\
\hline & & GCA & 10 & 2 & - & - & - & - & - & - & 1 & GTA & 1 ts & \\
\hline \multirow[t]{2}{*}{65} & K65R & AAA & 96 & 96 & 5 & 96 & 100 & 97 & 100 & 100 & 92 & AGA & 1 ts & 0.97 \\
\hline & & AAG & 4 & 3 & 95 & - & - & 3 & - & - & 6 & AGG & $1 \mathrm{ts}$ & \\
\hline \multirow[t]{2}{*}{67} & D67N & GAC & 70 & 90 & 86 & 70 & 97 & 90 & 26 & 93 & 13 & $\mathrm{AAC}$ & 1 ts & 0.99 \\
\hline & & GAT & 27 & 8 & 10 & 22 & 3 & 10 & 74 & 6 & 86 & AAT & 1 ts & \\
\hline \multirow[t]{2}{*}{69} & T69D & $\mathrm{ACT}$ & 97 & 95 & 76 & 93 & 100 & 90 & 26 & 5 & 94 & GAT & $1 \mathrm{ts}, 1 \mathrm{tv}$ & 0.20 \\
\hline & & $\mathrm{ACC}$ & - & 1 & 17 & 4 & - & 10 & 74 & 85 & 2 & GAC & $1 \mathrm{ts}, 1 \mathrm{tv}$ & \\
\hline \multirow[t]{2}{*}{70} & K70R & AAA & 84 & 95 & 15 & 15 & 100 & 95 & 93 & 100 & 98 & AGA & 1 ts & 1.00 \\
\hline & & AAG & 15 & 4 & 81 & 85 & - & 2 & 7 & - & 2 & AGG & 1 ts & \\
\hline \multirow[t]{3}{*}{74} & L74V & TTA & 90 & 93 & 97 & 93 & 100 & 40 & 96 & 96 & 94 & GTA & $1 \mathrm{tv}$ & 1.00 \\
\hline & & TTG & 1 & 3 & 1 & - & - & 57 & 4 & - & - & GTG & $1 \mathrm{tv}$ & \\
\hline & & CTA & 6 & 3 & 1 & 4 & - & - & - & 2 & 3 & GTA & $1 \mathrm{tv}$ & \\
\hline \multirow[t]{3}{*}{75} & V75I & GTA & 93 & 98 & 99 & 93 & 100 & 99 & 19 & 93 & 98 & ATA & $1 \mathrm{ts}$ & 0.05 \\
\hline & & GTT & 1 & - & - & - & - & - & 81 & 6 & - & ATT & $1 \mathrm{ts}$ & \\
\hline & & GTG & 4 & 1 & 1 & 7 & - & - & - & - & - & ATA & 2 ts & \\
\hline \multirow[t]{2}{*}{77} & F77L & TTC & 84 & 90 & 86 & 89 & 93 & 93 & 7 & 89 & 96 & CTC & $1 \mathrm{ts}$ & 0.97 \\
\hline & & TTT & 14 & 8 & 8 & 4 & 7 & 5 & 93 & 4 & 4 & CTT & $1 \mathrm{ts}$ & \\
\hline \multirow{2}{*}{115} & Y115F & TAT & 93 & 94 & 94 & 89 & 100 & 36 & 96 & 98 & 98 & TTT & $1 \mathrm{tv}$ & 1.00 \\
\hline & & TAC & 7 & 5 & 4 & 11 & - & 62 & 4 & 2 & 1 & TTC & $1 \mathrm{tv}$ & \\
\hline \multirow[t]{2}{*}{116} & F116Y & TTT & 96 & 90 & 67 & 89 & 90 & 97 & 100 & 96 & 96 & TAT & $1 \mathrm{tv}$ & 0.99 \\
\hline & & TTC & 1 & 10 & 31 & 8 & 10 & 1 & - & 4 & 3 & TAC & $1 \mathrm{tv}$ & \\
\hline \multirow[t]{4}{*}{118} & V118I & GTT & 80 & 92 & 86 & 78 & 90 & 25 & 74 & 93 & 79 & ATT & 1 ts & $<0.001$ \\
\hline & & GTC & 8 & 2 & 3 & - & 3 & 7 & 22 & 6 & 10 & ATC & $1 \mathrm{ts}$ & \\
\hline & & GTA & 3 & 1 & 1 & 7 & 3 & 3 & - & - & 1 & ATA & 1 ts & \\
\hline & & GTG & 1 & 1 & 3 & - & 3 & 63 & - & 2 & 6 & ATA & 2 ts & \\
\hline 151 & Q151M & CAG & 86 & 91 & 91 & 59 & 13 & 93 & 96 & 98 & 93 & ATG & $2 \mathrm{tv}$ & $<0.001$ \\
\hline & & CAA & 12 & 7 & 5 & 37 & 83 & 6 & 4 & 2 & 4 & ATG & $1 \mathrm{ts}, 2 \mathrm{tv}$ & \\
\hline 184 & M184I & ATG & 100 & 100 & 100 & 100 & 100 & 100 & 100 & 100 & 100 & ATA & 1 ts & 0.97 \\
\hline & M184V & ATG & & & & & & & & & & GTG & 1 ts & 0.97 \\
\hline 210 & L210W & TTG & 82 & 85 & 65 & 89 & 13 & 9 & 82 & 87 & 11 & TGG & $1 \mathrm{tv}$ & $<0.001$ \\
\hline & & TTA & 7 & 9 & 29 & 4 & 7 & 63 & 4 & 9 & 11 & TGG & $1 \mathrm{ts}, 1 \mathrm{tv}$ & \\
\hline & & CTG & 6 & 2 & 1 & 4 & 43 & 19 & 7 & 2 & 72 & TGG & $1 \mathrm{ts}, 1 \mathrm{tv}$ & \\
\hline & & CTA & - & - & - & - & 23 & 2 & - & - & 6 & TGG & $2 \mathrm{ts}, 1 \mathrm{tv}$ & \\
\hline 215 & $\mathrm{~T} 215 \mathrm{~F}$ & $\mathrm{ACC}$ & 8 & 96 & 92 & 93 & 43 & 96 & 96 & 4 & 89 & TTC & $1 \mathrm{ts}, 1 \mathrm{tv}$ & 0.41 \\
\hline & & $\mathrm{ACT}$ & 88 & 4 & 3 & - & 53 & 2 & - & 96 & 10 & TTT & $1 \mathrm{ts}, 1 \mathrm{tv}$ & \\
\hline & $\mathrm{T} 215 \mathrm{Y}$ & $\mathrm{ACC}$ & & & & & & & & & & TAC & $2 \mathrm{tv}$ & 0.37 \\
\hline & & $\mathrm{ACT}$ & & & & & & & & & & TAT & $2 \mathrm{tv}$ & \\
\hline 219 & K219E & AAA & 96 & 93 & 17 & 100 & 87 & 93 & 96 & 100 & 92 & GAA & 1 ts & 0.81 \\
\hline & & AAG & 3 & 5 & 80 & - & 10 & 7 & - & - & 4 & GAG & $1 \mathrm{ts}$ & \\
\hline & K219Q & AAA & & & & & & & & & & CAA & $1 \mathrm{tv}$ & 0.60 \\
\hline & & AAG & & & & & & & & & & CAG & $1 \mathrm{tv}$ & \\
\hline
\end{tabular}

*ts indicates transition; tv, transversion.

$\dagger P$ value calculated using the Kruskal-Wallis test and a value of 2.5 for a transversion and 1 for a transition. For statistical reasons, a random sample of $20 \%$ of the subtype B and all sequences classified with a subtype other than B were included in the Kruskal-Wallis test.

†umber of sequences between brackets.

§RF01_AE.

|CRF02_AG. 
TABLE 4. Prevalence of Wild-Type (WT) Codons and Its Impact on Calculated Genetic Barrier at NNRTI Resistance-Associated Positions in Anti-Retroviral-Naive Patients

\begin{tabular}{|c|c|c|c|c|c|c|c|c|c|c|c|c|c|c|}
\hline \multirow[b]{2}{*}{ Codon } & \multirow[b]{2}{*}{ Substitution } & \multirow[b]{2}{*}{$\begin{array}{c}\text { WT } \\
\text { Codon }\end{array}$} & \multicolumn{9}{|c|}{ Proportion (\%) WT Codon by HIV-1 Subtype } & \multirow[b]{2}{*}{$\begin{array}{c}\text { Resistant } \\
\text { Codon }\end{array}$} & \multirow[b]{2}{*}{ Mutation* } & \multirow[b]{2}{*}{$P \dagger$} \\
\hline & & & $\begin{array}{c}\text { A } \\
\text { (73) } \ddagger\end{array}$ & $\begin{array}{c}\text { B } \\
(\mathbf{1 3 3 8})\end{array}$ & $\begin{array}{c}C \\
(213)\end{array}$ & $\begin{array}{c}\text { D } \\
\text { (27) }\end{array}$ & $\begin{array}{c}F \\
(30)\end{array}$ & $\begin{array}{c}\text { G } \\
(89)\end{array}$ & $\begin{array}{c}\mathbf{J} \\
(27)\end{array}$ & $\begin{array}{l}\mathrm{AE} \S \\
(\mathbf{5 5})\end{array}$ & $\begin{array}{l}\mathbf{A G} \| \\
\mathbf{( 9 0 )}\end{array}$ & & & \\
\hline \multirow[t]{4}{*}{100} & L100I & TTA & 49 & 87 & 92 & 22 & 80 & 90 & 96 & 91 & 90 & ATA & $1 \mathrm{tv}$ & 0.01 \\
\hline & & CTA & 34 & 6 & 1 & $63 \S$ & 13 & 4 & 4 & 5 & 4 & ATA & $1 \mathrm{tv}$ & \\
\hline & & TTG & 8 & - & - & - & 3 & 4 & - & 2 & 2 & ATA & $1 \mathrm{ts}, 1 \mathrm{tv}$ & \\
\hline & & CTG & 1 & - & - & 15 & - & - & - & - & - & ATA & $1 \mathrm{ts}, 1 \mathrm{tv}$ & \\
\hline \multirow[t]{2}{*}{103} & K103N & AAA & 95 & 91 & 93 & 100 & 93 & 99 & 96 & 91 & 97 & $\mathrm{AAC} / \mathrm{T}$ & $1 \mathrm{tv}$ & 0.35 \\
\hline & & AAG & 1 & 5 & 3 & - & 3 & 1 & - & 7 & 2 & $\mathrm{AAC} / \mathrm{T}$ & $1 \mathrm{tv}$ & \\
\hline \multirow[t]{4}{*}{106} & V106A & GTA & 97 & 93 & 12 & 85 & 100 & 98 & 85 & 89 & 97 & GCA & 1 ts & 0.15 \\
\hline & & GTG & - & 2 & 83 & - & - & 1 & 4 & 7 & - & GCG & 1 ts & \\
\hline & V106M & GTA & & & & & & & & & & ATG & 2 ts & $<0.001$ \\
\hline & & GTG & & & & & & & & & & ATG & $1 \mathrm{ts}$ & \\
\hline \multirow[t]{2}{*}{108} & V108I & GTA & 89 & 91 & 91 & 96 & 93 & 35 & 100 & 98 & 88 & ATA & 1 ts & $<0.001$ \\
\hline & & GTG & 5 & 2 & 6 & - & 7 & 62 & - & - & 4 & ATA & 2 ts & \\
\hline \multirow[t]{4}{*}{181} & Y181C & TAT & 89 & 93 & 96 & 93 & 13 & 11 & 74 & 100 & 16 & TGT & 1 ts & 0.80 \\
\hline & & TAC & 5 & 5 & 1 & 4 & 83 & 88 & 15 & - & 81 & TGC & $1 \mathrm{ts}$ & \\
\hline & Y181I & TAT & & & & & & & & & & ATT & $2 \mathrm{tv}$ & 1.00 \\
\hline & & TAC & & & & & & & & & & ATC & $2 \mathrm{tv}$ & \\
\hline \multirow[t]{3}{*}{188} & Y188C & TAT & 100 & 99 & 100 & 96 & 100 & 100 & 100 & 96 & 96 & TGT & 1 ts & 0.97 \\
\hline & Y188H & TAT & & & & & & & & & & CAT & $1 \mathrm{ts}$ & 0.97 \\
\hline & Y188L & TAT & & & & & & & & & & TTA & $2 \mathrm{tv}$ & 0.07 \\
\hline \multirow[t]{6}{*}{190} & G190A & GGA & 86 & 90 & 95 & 89 & 7 & 91 & 96 & 100 & 93 & GCA & $1 \mathrm{tv}$ & 0.15 \\
\hline & & GGC & 8 & 3 & 1 & 7 & 7 & 8 & - & - & 2 & GCC & $1 \mathrm{tv}$ & \\
\hline & & GGG & 5 & 4 & - & - & 87 & - & - & - & 2 & GCG & $1 \mathrm{tv}$ & \\
\hline & G190S & GGA & & & & & & & & & & AGC/TC & $1 \mathrm{ts}, 1 \mathrm{tv}$ & 0.16 \\
\hline & & GGC & & & & & & & & & & AGC & 1 ts & \\
\hline & & GGG & & & & & & & & & & $\mathrm{AGC} / \mathrm{T}$ & $1 \mathrm{ts}, 1 \mathrm{tv}$ & \\
\hline \multirow[t]{4}{*}{2259} & $\mathrm{P} 225 \mathrm{H}$ & $\mathrm{CCT}$ & 71 & 80 & 30 & 72 & 36 & 86 & 67 & 93 & 90 & CAT & $1 \mathrm{tv}$ & $<0.001$ \\
\hline & & $\mathrm{CCC}$ & 10 & 13 & 64 & 17 & 64 & 10 & 33 & 7 & 8 & CAC & $1 \mathrm{tv}$ & \\
\hline & & $\mathrm{CCA}$ & 6 & 1 & 1 & - & - & 2 & - & - & - & $\mathrm{CAC} / \mathrm{T}$ & $2 \mathrm{tv}$ & \\
\hline & & CCG & 9 & 1 & 1 & - & - & - & - & - & - & $\mathrm{CAC} / \mathrm{T}$ & $2 \mathrm{tv}$ & \\
\hline 230 वा & M230L & ATG & 100 & 100 & 100 & 100 & 100 & 100 & 100 & 100 & 100 & $\mathrm{C} / \mathrm{TTG}$ & $1 \mathrm{tv}$ & 1.00 \\
\hline 2369 & $\mathrm{P} 236 \mathrm{~L}$ & $\mathrm{CCT}$ & 100 & 100 & 100 & 100 & 100 & 100 & 100 & 100 & 100 & CTT & $1 \mathrm{tv}$ & 1.00 \\
\hline \multicolumn{15}{|c|}{$\begin{array}{l}\text { *ts indicates transition; tv, transversion. } \\
\dagger P \text { value calculated using the Kruskal-Wall } \\
\text { all sequences classified with a subtype other the } \\
\text { †Number of sequences between brackets. } \\
\text { §CRF01_AE. } \\
\| \text { CRF02_AG. }\end{array}$} \\
\hline
\end{tabular}

protease $(70 \%)$ and $1338 \mathrm{RT}$ sequences $(69 \%)$. A total of 556 protease and 604 RT sequences were classified as a non-B subtype. Among these sequences, subtype $\mathrm{C}$ was the most common subtype (11\%).

\section{Protease Inhibitor-Associated Resistance Major Substitutions}

Most codons were remarkably conserved among the subtypes on positions with major protease substitutions (Table 2). Interestingly, only at position 82 a codon was identified that did make an impact on the genetic barrier. Here, most subtype G sequences had an isoleucine (ATC) compared with valine (GTC) in other subtypes. The shortest distance to a resistance substitution in subtype $\mathrm{G}$ would be the I82T substitution (facilitated by 1 transition from ATC to $\mathrm{ACC}$ ), where other subtypes could likewise evolve to V82A by a single transition from GTC to GCC. The ATC polymorphism at position 82 was also found in a small proportion of all other subtypes, except A and F. The ATC polymorphism also affected the calculated genetic barrier across the subtypes for substitutions I/V82A and I/V82T $(P<$ $0.001)$. For the statistical comparison of the calculated genetic barrier for I/V82A, a score of 1 was used for $96 \%$ of subtype $B$ and $14 \%$ of subtype $G$ sequences (these sequences required a single transition), and a score of 2 was used for $1 \%$ of subtype $B$ and $84 \%$ of subtype $G$ (the sequences needed 2 transitions). Using these scores, an 
TABLE 5. Results of the Statistical Comparisons of the Calculated Genetic Barrier for Substitutions Where a Different Genetic Barrier Was Calculated Between the Subtypes in Tables 2, 3, and 4

\begin{tabular}{|c|c|c|c|c|c|c|c|c|}
\hline \multirow[b]{2}{*}{ Mutation* } & \multicolumn{8}{|c|}{ Subtype $\dagger$} \\
\hline & $\mathbf{A}$ & $\mathrm{C}$ & D & $\mathbf{F}$ & G & $\mathbf{J}$ & CRF_AE & CRF_AG \\
\hline \multicolumn{9}{|l|}{ PI } \\
\hline V82A & 0.608 & $<0.001$ & 0.035 & 0.751 & $<0.001$ & 0.019 & 0.020 & 0.009 \\
\hline V82T & 0.609 & $<\mathbf{0 . 0 0 1}$ & 0.468 & 0.476 & $<\mathbf{0 . 0 0 1}$ & 0.363 & 0.024 & 0.676 \\
\hline \multicolumn{9}{|l|}{ NRTI } \\
\hline V75I & 0.177 & 0.567 & 0.043 & 0.497 & 0.243 & 0.519 & 0.074 & 0.786 \\
\hline V118I & 0.997 & 0.470 & 0.009 & 0.375 & $<0.001$ & 0.348 & 0.513 & 0.010 \\
\hline Q151M & 0.085 & 0.671 & $<0.001$ & $<\mathbf{0 . 0 0 1}$ & 0.873 & 0.633 & 0.225 & 0.575 \\
\hline L210W & 0.113 & $<\mathbf{0 . 0 0 1}$ & 0.500 & $<\mathbf{0 . 0 0 1}$ & $<0.001$ & 0.532 & 0.213 & $<0.001$ \\
\hline \multicolumn{9}{|l|}{ NNRTI } \\
\hline L100I & 0.295 & 0.010 & 0.089 & 0.542 & 0.576 & 0.188 & 0.202 & 0.151 \\
\hline V106M & 0.166 & $<\mathbf{0 . 0 0 1}$ & 0.732 & 0.230 & 0.233 & 0.024 & 0.200 & 0.418 \\
\hline V108I & 0.158 & 0.035 & 0.429 & 0.166 & $<0.001$ & 0.429 & 0.259 & 0.290 \\
\hline $\mathrm{P} 225 \mathrm{H}$ & $<0.001$ & 0.231 & 0.354 & 0.288 & 0.514 & 0.553 & 0.471 & 0.084 \\
\hline
\end{tabular}

*Only mutations with $P<0.05$ in Tables 1,2 , and 3 were considered as for these mutations a differential calculated genetic barrier was assessed.

$\dagger$ Numbers are $P$ values comparing the calculated genetic barrier between an individual non-B and subtype B. $P$ values were determined using the Mann-Whitney $U$ test. Values in bold were statistically significant $(P<0.001$ was required for significant differences because of the Benjamini-Hochberg method to adjust for multiple hypothesis).

increased genetic barrier was found for subtype $\mathrm{G}(P<$ 0.001). Similarly, subtype $\mathrm{C}$ also had an increased calculated genetic barrier for $82 \mathrm{~A}$ compared with subtype $\mathrm{B}(P<0.001)$. Furthermore, a decreased genetic barrier was found for $82 \mathrm{~T}$ $(P<0.001)$ in subtypes $\mathrm{C}$ and $\mathrm{G}$ versus subtype $\mathrm{B}(P<0.001)$ (Table 5).

\section{Minor Protease Substitutions}

The average number of minor protease substitutions showed an extensive variation between the subtypes (Table 6). Notably, limited polymorphism (present in $<2 \%$ in any particular subtype) was found for $\mathrm{L} 10 \mathrm{~F} / \mathrm{R}, \mathrm{K} 20 \mathrm{M} / \mathrm{L} / \mathrm{T} / \mathrm{V}$, L24I, V32I, E35G, K43T, I47A/V, F53L, I54V/L/A/M/T/S, Q58E, G73C/S/T/A, T74P, N83D, and N88D/S. Similarly, polymorphism (present $>2 \%$ in any subtype) was found for $\mathrm{L} 10 \mathrm{I} / \mathrm{V}, \mathrm{I} 13 \mathrm{~V}(>80 \%$ in subtypes $\mathrm{A}, \mathrm{G}$, CRF01_AE, and CRF02_AG), K20I ( $>90 \%$ in subtypes G and CRF02_AG), K20R, L33F, M36I ( $>70 \%$ in all non-B subtypes, $17 \%$ in B), L63P, H69K (>90\% in subtypes A, C, G, J, CRF01_AE, and CRF02_AG), and V77I.

The sequences of all individual non-B subtypes contained on average more minor protease substitutions relevant for indinavir, nelfinavir, and ritonavir. This increased number was statistically significant for all particular non-B subtypes $(P<0.001)$, except for subtype D. Similarly, all particular non-B subtypes had a relatively larger number of substitutions relevant for atazanavir and tipranavir $(P<$ 0.001). A notable diversity was found for amprenavir. Sequences classified as subtype A or B had on average the largest number of minor substitutions relevant for this protease inhibitor. Comparing the individual non-B subtypes to subtype B only revealed a significant result for subtypes $\mathrm{C}$ (increased genetic barrier, $P<0.001$ ) and A (decreased genetic barrier, $P=0.001)$.

TABLE 6. Results of the Statistical Comparison of the Mean Ratio of the Number of Minor Protease Substitutions in a Particular Non-B Subtype and Subtype B

\begin{tabular}{|c|c|c|c|c|c|c|c|c|}
\hline \multirow[b]{2}{*}{ Protease Inhibitor } & \multicolumn{8}{|c|}{ Subtype } \\
\hline & $\mathbf{A}$ & $\mathbf{C}$ & D & $\mathbf{F}$ & G & $\mathbf{J}$ & CRF AE & CRF AG \\
\hline Amprenavir & $2.2 *(\mathbf{0 . 0 0 1}) \dagger$ & $0.3(<0.001) \dagger$ & $0.6(0.44)$ & $0.3(0.15)$ & $0.5(0.09)$ & $0.4(0.26)$ & $1.1(0.78)$ & $0.4(0.06)$ \\
\hline Atazanavir & $3.5(<\mathbf{0 . 0 0 1})$ & $2.6(<\mathbf{0 . 0 0 1})$ & $2.4(<\mathbf{0 . 0 0 1})$ & $3.3(<\mathbf{0 . 0 0 1})$ & $4.8(<\mathbf{0 . 0 0 1})$ & $3.0(<\mathbf{0 . 0 0 1})$ & $3.2(<\mathbf{0 . 0 0 1})$ & $4.8(<\mathbf{0 . 0 0 1})$ \\
\hline Indinavir & $2.0(<\mathbf{0 . 0 0 1})$ & $1.6(<\mathbf{0 . 0 0 1})$ & $1.6(0.02)$ & $2.1(<\mathbf{0 . 0 0 1})$ & $1.6(<\mathbf{0 . 0 0 1})$ & $2.5(<\mathbf{0 . 0 0 1})$ & $1.8(<\mathbf{0 . 0 0 1})$ & $1.5(<\mathbf{0 . 0 0 1})$ \\
\hline Lopinavir/ritonavir & $0.9(0.58)$ & $0.7(<0.001)$ & $0.8(0.13)$ & $0.7(0.06)$ & $0.2(<0.001)$ & $0.6(0.001)$ & $0.6(<0.001)$ & $0.2(<0.001)$ \\
\hline Nelfinavir & $1.8(<\mathbf{0 . 0 0 1})$ & $1.4(<\mathbf{0 . 0 0 1})$ & $1.3(0.09)$ & $1.6(<\mathbf{0 . 0 0 1})$ & $1.6(<\mathbf{0 . 0 0 1})$ & $2.4(<\mathbf{0 . 0 0 1})$ & $1.6(<\mathbf{0 . 0 0 1})$ & $1.4(<\mathbf{0 . 0 0 1})$ \\
\hline Ritonavir & $2.0(<\mathbf{0 . 0 0 1})$ & $1.6(<\mathbf{0 . 0 0 1})$ & $1.6(0.02)$ & $2.1(<\mathbf{0 . 0 0 1})$ & $1.6(<\mathbf{0 . 0 0 1})$ & $2.6(<\mathbf{0 . 0 0 1})$ & $1.8(<\mathbf{0 . 0 0 1})$ & $1.5(<\mathbf{0 . 0 0 1})$ \\
\hline Saquinavir & $0.6(0.03)$ & $0.2(<0.001)$ & $0.5(0.05)$ & $0.4(0.01)$ & $0.3(<0.001)$ & $1.4(0.04)$ & $0.3(<0.001)$ & $0.1(<\mathbf{0 . 0 0 1})$ \\
\hline Tipranavir & $7.4(<\mathbf{0 . 0 0 1})$ & $5.1(<\mathbf{0 . 0 0 1})$ & $4.2(<\mathbf{0 . 0 0 1})$ & $3.9(<\mathbf{0 . 0 0 1})$ & $7.1(<\mathbf{0 . 0 0 1})$ & $5.3(<\mathbf{0 . 0 0 1})$ & $7.4(<\mathbf{0 . 0 0 1})$ & $7.4(<\mathbf{0 . 0 0 1})$ \\
\hline All minor mutations & $1.4(<\mathbf{0 . 0 0 1})$ & $1.2(\mathbf{0 . 0 0 2})$ & $1.2(0.48)$ & $1.3(0.02)$ & $1.7(<\mathbf{0 . 0 0 1})$ & $1.7(<\mathbf{0 . 0 0 1})$ & $1.2(0.09)$ & $1.7(<\mathbf{0 . 0 0 1})$ \\
\hline
\end{tabular}


Subtype B sequences contained the highest frequency of minor protease substitutions relevant for lopinavir/ ritonavir. Statistical significance was, however, only reached for subtypes B versus C, G, J, CRF01_AE, and CRF02_AG (all $P<0.001$ ). In the same way, subtype $\mathrm{B}$ had significantly more minor protease substitutions relevant for saquinavir versus subtypes C, G, CRF01_AE, and CRF02_AG (all $P<0.001)$.

\section{NRTI Resistance-Associated Substitutions}

Synonymous differences were common across subtypes at nucleoside reverse transcription inhibitor (NRTI) resistance-related positions (Table 3). Interestingly, these synonymous differences at the nucleotide level did not affect, in general, the genetic barrier for evolution to NRTI resistance-associated substitutions. The synonymous differences in codon usage did only have an impact for 3 codons: V118I $(P<0.001)$, Q151M $(P<0.001)$, and L210W $(P<0.001)$.

For the V118I substitution, 2 transitions are necessary for a majority of subtype $\mathrm{G}$ to change $G \mathrm{~T} G$ to $A \mathrm{~T} A$, whereas for most other subtypes only a single transition was needed ( $G \mathrm{TT}$ to $A \mathrm{TT} ; P<0.001$ ).

Similarly, most subtype $\mathrm{F}$ sequences and a substantial number of subtype D sequences have an increased genetic barrier for the Q151M substitution. In these particular subtypes, 2 transversions and 1 transition are required to mutate the wild-type codon $C A A$ to $A T G$. This contrasts to other subtypes where only 2 transversions are generally needed ( $C A \mathrm{G}$ to $A T \mathrm{G} ; P<0.001)$.

Most subtype C, F, G, and CRF02_AG sequences have an increased genetic barrier for evolution to the L210W substitution. Strains classified with these subtypes contain either the CTG or the TTA polymorphism at this position. Both the CTG and TTA codon facilitate the L210W substitution by 1 transition and 1 transversion (CTG or TTA to $T G \mathrm{G}, P<0.001)$. Other subtypes generally require 1 transition less to mutate codon 210 from TTG to TGG.

\section{NNRTI Resistance-Associated Substitutions}

All subtypes contained the same amino acids at all NNRTI resistance-related positions. At several positions, a few subtypes could be distinguished by synonymous differences at the nucleotide level. These synonymous differences did only affect the calculated genetic barrier for substitutions V106M, V108I, and P225H (all $P<0.001$, Table 4).

The previously reported codon GTG at position 106 of subtype $C,{ }^{3}$ which has a reduced genetic barrier for V106M, was also found in this study. Interestingly, the GTG codon also occurred in a few other subtypes including B. This suggests that V106M can occur at low incidence in subtypes other than $\mathrm{C}$.

For the V108I substitution, a majority of subtype $\mathrm{G}$ sequences required 2 transitions to substitute $G \mathrm{~T} G$ to $A \mathrm{~T} A$, which is 1 additional transition compared with other subtypes that only need a single transition to evolve from $G$ TA to $A$ TA $(P<0.001)$.

Finally, at position 225, a minority of subtype A sequences contained the codon $\mathrm{C} C A$ or $\mathrm{C} C G$ (proline), which requires 2 transversions for evolution to the drug-resistanceassociated substitution $\mathrm{C} A T$ or $\mathrm{C} A C$ (histidine). Subtype A has an increased genetic barrier compared with other subtypes, which contained a CCT or CCC codon that required only a single transversion $(P<0.001$ compared with subtype $\mathrm{B})$.

\section{Geographical Differences}

The codons associated with a differential calculated genetic barrier were compared between countries. Compared to subtype $\mathrm{G}$ sequences from other countries in Europe, the Portuguese subtype $G$ sequences included a relatively larger proportion of polymorphisms associated with an increased genetic barrier at RT positions 108, 118, and 210. Other intersubtype geographical differences were not found (data not shown).

\section{DISCUSSION}

The genetic barrier is an important factor for the development of HIV drug resistance. Because of huge genetic variability in HIV-1, particular subtypes could have different genetic barriers for drug resistance substitutions. In the present study, nearly 2000 protease and RT gene sequences ( $>600$ non-B) obtained from anti-retroviral-naive patients were compared for differences between HIV-1 subtypes in the calculated genetic barrier. Remarkably, the genetic barrier was similar for all subtypes at almost all positions. In addition, for the few positions where differences were found, a higher genetic barrier was frequently calculated for some individual non-B subtypes.

In protease, a different genetic barrier was only computed for $82 \mathrm{~A}$ and $82 \mathrm{~T}$ (increased and decreased genetic barrier in subtypes $\mathrm{C}$ and $\mathrm{G}$, respectively). In general, 82A and $82 \mathrm{~T}$ have a comparable impact on protease inhibitor susceptibility. ${ }^{7}$

Extensive differences between the subtypes were found for the minor protease substitutions. Importantly, in vitro studies have shown that minor protease substitutions do not impair drug susceptibility, but that they affect the genetic pathway of resistance likely by modulating the fitness and resistance once the virus generates a relevant major substitution. ${ }^{14,15}$ Minor protease substitutions may influence resistance pathways, which could explain that some subtypes selected D30N and others L90M under nelfinavir exposure. ${ }^{16,17}$ In this respect, the findings from this study provide important data, as it can be expected that drug resistance to a particular protease inhibitor is enhanced in some subtypes.

The NRTI resistance-associated substitutions where an increased genetic barrier was found included V118I in subtype $G$ sequences, Q151M in subtypes $F$ and $D$, and L210W in subtypes C, F, G, and CRF02_AG. The V118I substitution occurring in isolation has probably no significant impact on drug susceptibility. More important is the Q151M substitution, which is part of a multi-NRTI resistance complex that is associated with resistance to all NRTIs. ${ }^{7}$

For NNRTI resistance-related substitutions, a differential genetic barrier was found for V106M (reduced genetic barrier in subtype C), V108I (increased genetic barrier in subtype G), and P225H (increased genetic barrier in subtype 
A). The V106M substitution confers high-level resistance to all NNRTIs. ${ }^{3}$ V108I and P225H each contribute to efavirenz resistance when present in combination with other NNRTIassociated substitutions, but occurring alone they do not confer measurable in vitro. ${ }^{7}$

An explanation why so few major differences were found is difficult to give. A possible explanation is that antiretroviral drugs target sites that are functionally important and which are therefore well conserved across the subtypes. The functional importance is highlighted by the reduced fitness found in many sequences containing major drugresistance-associated substitutions. ${ }^{18}$ Finally, although the amino acids are fairly well conserved across the subtypes, it cannot be excluded that for subtypes $\mathrm{D}, \mathrm{F}$, and $\mathrm{J}$ relevant differences were missed because of their low numbers.

The $\mathrm{G} \rightarrow$ A hypermutation ${ }^{19}$ is the most frequently occurring nucleotide mutation in HIV $-1 .^{20,21}$ The model used for calculating the genetic barrier did not account for the $G \rightarrow$ A hypermutation. But comparison of the wild-type and the drug resistance codons shows that this hypermutation does not contribute to a differential genetic barrier between any subtype. Not including the $\mathrm{G} \rightarrow \mathrm{A}$ has therefore not impacted on the results.

The genetic barrier was computed using a transitiontransversion bias of 2.5. Importantly, the appropriate statistical tests for comparison of the calculated genetic barrier the Kruskal-Wallis and Mann-Whitney $U$ test - are based on rank order. ${ }^{22}$ The rank orders are not influenced by the absolute value of the transition-transversion bias. As a consequence, choosing another value for the transitiontransversion bias would have led to similar results.

The genetic barrier is an important determinant for emergence of drug resistance. Other factors, however, also contribute to the development of resistance. These include viral (eg, replication capacity of the drug-resistant virus, genetic background, host cell tropism, mutations outside protease and RT) and host factors (eg, immune control and target cell availability). ${ }^{18}$

In addition, this study considered the substitutions as defined by the IAS. ${ }^{7}$ These substitutions were predominantly identified in subtype B viruses. Drug-resistance-associated amino acid substitutions that might be identified in the future as being relevant for non-B subtypes could not be considered.

The importance of some of the positions and subtypes with a differential genetic barrier has been reported previously. $^{23,24}$ These studies included a smaller number of subtypes, did not quantify the genetic barrier, and omitted the minor protease substitutions. Dumans et $\mathrm{al}^{23}$ analyzed the influence of synonymous genetic polymorphisms mutational routes to drug resistance in a smaller number of Brazilian treatment-naive patients that were limited to subtypes B, C, and F1 sequences. Interestingly, they also found that subtype $\mathrm{F}$ has a higher genetic barrier to acquire the Q151M and the L210W substitutions as compared with subtype B. ${ }^{23}$

Turner et $\mathrm{al}^{24}$ compared nucleotide mutations and polymorphisms at codons known to confer resistance in subtype B. Their findings also identified the GTG polymorphism at RT position 106 of subtype $\mathrm{C}$ and the expected selection of I82T substitution in subtype G. Unfortunately, because of small numbers of sequences, differential genetic barriers were not identified in several subtypes. ${ }^{24}$

Clinical studies that investigated the antiviral effect of different regimens have not revealed any clear association between HIV subtype and virological response. ${ }^{25,26}$ A first report compared virological response to therapy in 479 therapy-naive patients, including $4.4 \%$ infected with HIV of a non-B subtype who were starting treatment in Canada. It was found that the virological response was independent of subtype. $^{25}$ Another study also found no evidence of differential virological response in 113 children infected with viruses of different subtype in Europe. ${ }^{26}$

In conclusion, this study found only limited differences between subtypes in the calculated genetic barrier for evolution to major drug-resistance-associated substitutions. These results imply that subtype diversity is of minor influence on the patterns of resistance substitutions that will emerge. Particular subtypes could, however, enhance protease inhibitor resistance due to the presence of a larger number of relevant minor substitutions. The results of this study are of the utmost importance for the clinical management of the increasing number of patients across the World that receive treatment of HIV.

\section{ACKNOWLEDGEMENTS}

This work resulted from the SPREAD project that was supported by the European Commission (contract number QLK2-CT-2001-01344).Part of this study was presented at the XIII international HIV drug resistance workshop that was held June 8-12, 2004, in Tenerife, Canary Islands, Spain (abstract 87). The authors gratefully acknowledge Jan Albert, Viktor Müller, and Monique Nijhuis for critical review of the paper.

\section{REFERENCES}

1. Kuritzkes DR. Preventing and managing resistance in the clinical setting. J Acquir Immune Defic Syndr. 2003;34(Suppl 2):S103-S110.

2. Beerenwinkel N, Daumer M, Sing T, et al. Estimating HIV evolutionary pathways and the genetic barrier to drug resistance. J Infect Dis. 2005; 191(11):1953-1960.

3. Brenner B, Turner D, Oliveira M, et al. A V106M mutation in HIV-1 clade $\mathrm{C}$ viruses exposed to efavirenz confers cross-resistance to non-nucleoside reverse transcriptase inhibitors. AIDS. 2003;17(1):F1-F5.

4. Parkin NT, Schapiro JM. Antiretroviral drug resistance in non-subtype B HIV-1, HIV-2 and SIV. Antivir Ther. 2004;9(1):3-12.

5. Grossman Z, Istomin V, Averbuch D, et al. Genetic variation at NNRTI resistance-associated positions in patients infected with HIV-1 subtype C. AIDS. 2004;18(6):909-915.

6. Wensing AM, van de Vijver DA, Angarano G, et al. Prevalence of drugresistant HIV-1 variants in untreated individuals in Europe: implications for clinical management. J Infect Dis. 2005;192(6):958-966.

7. Johnson VA, Brun-Vezinet F, Clotet B, et al. Update of the drug resistance mutations in HIV-1: 2005. Top HIV Med. 2005;13(1):51-57.

8. Nijhuis M, Schuurman R, de Jong D, et al. Increased fitness of drug resistant HIV-1 protease as a result of acquisition of compensatory mutations during suboptimal therapy. AIDS. 1999;13(17):2349-2359.

9. Grossman Z, Vardinon N, Chemtob D, et al. Genotypic variation of HIV-1 reverse transcriptase and protease: comparative analysis of clade C and clade B. AIDS. 2001;15(12):1453-1460.

10. Wu TD, Schiffer CA, Gonzales MJ, et al. Mutation patterns and structural correlates in human immunodeficiency virus type 1 protease following different protease inhibitor treatments. J Virol. 2003;77(8): 4836-4847. 
11. de Oliveira T, Deforche K, Cassol S, et al. An automated genotyping system for analysis of HIV-1 and other microbial sequences. Bioinformatics. 2005;21(19):3797-3800.

12. Vandamme AM. Basic concepts of molecular evolution. In: Salemi M, Vandamme AM, eds. The Phylogenetic Handbook. A Practical Approach to DNA and Protein Phylogeny. Cambridge: Cambridge University Press, 2004.

13. Benjami Y, Hochberg Y. Controlling the false discovery rate: a practical and powerful approach to multiple testing. J R Stat Soc B. 1995;57: 289-300.

14. Gonzalez LMF, Brindeiro RM, Aguiar RS, et al. Impact of nelfinavir resistance mutations on in vitro phenotype, fitness and replication capacity of HIV-1 with subtype B and C proteases. Antivir Ther. 2004; 9:S65.

15. Qari SH, Pieniazek D, Heneine W. Resistance-related polymorphisms in HIV-1 non-B subtype protease influence the resistance pathway and amplify resistance to protease inhibitors. Antivir Ther. 2004; 9:S45.

16. Grossman Z, Paxinos EE, Averbuch D, et al. Mutation D30N is not preferentially selected by human immunodeficiency virus type 1 subtype $\mathrm{C}$ in the development of resistance to nelfinavir. Antimicrob Agents Chemother. 2004:48(6):2159-2165.

17. Cane PA, de Ruiter A, Rice P, et al. Resistance-associated mutations in the human immunodeficiency virus type 1 subtype c protease gene from treated and untreated patients in the United Kingdom. J Clin Microbiol. 2001;39(7):2652-2654.

18. Nijhuis M, Deeks S, Boucher C. Implications of antiretroviral resistance on viral fitness. Curr Opin Infect Dis. 2001;14(1):23-28.
19. Vartanian JP, Meyerhans A, Asjo B, et al. Selection, recombination, and $\mathrm{G}$ - a hypermutation of human immunodeficiency virus type 1 genomes. J Virol. 1991;65(4):1779-1788.

20. Leitner T, Kumar S, Albert J. Tempo and mode of nucleotide substitutions in gag and env gene fragments in human immunodeficiency virus type 1 populations with a known transmission history. $J$ Virol. 1997;71(6):4761-4770.

21. Bishop KN, Holmes RK, Sheehy AM, et al. APOBEC-mediated editing of viral RNA. Science. 2004;305(5684):645.

22. Bland M. An Introduction to Medical Statistics, 3rd ed. Oxford: Oxford University Press, 2000.

23. Dumans AT, Soares MA, Machado ES, et al. Synonymous genetic polymorphisms within Brazilian human immunodeficiency virus type 1 subtypes may influence mutational routes to drug resistance. J Infect Dis. 2004;189(7):1232-1238

24. Turner D, Brenner B, Moisi D, et al. Nucleotide and amino Acid polymorphisms at drug resistance sites in non-B-subtype variants of human immunodeficiency virus type 1. Antimicrob Agents Chemother. 2004;48(8):2993-2998.

25. Alexander CS, Montessori V, Wynhoven B, et al. Prevalence and response to antiretroviral therapy of non-B subtypes of HIV in antiretroviral-naive individuals in British Columbia. Antivir Ther. 2002; 7(1):31-35.

26. Pillay D, Walker AS, Gibb DM, et al. Impact of human immunodeficiency virus type 1 subtypes on virologic response and emergence of drug resistance among children in the Paediatric European Network for Treatment of AIDS (PENTA) 5 trial. $J$ Infect Dis. 2002;186(5):617-625. 